\title{
The ultraviolet spectra of active galaxies with double-peaked emission lines
}

\author{
M. Eracleous ${ }^{1}$, J. P. Halpern ${ }^{2}$, T. Storchi-Bergmann ${ }^{3}$, \\ A. V. Filippenko ${ }^{4}$, A. S. Wilson ${ }^{5}$ and M. Livio ${ }^{6}$ \\ ${ }^{1}$ Deptartment of Astronomy \& Astrophysics, The Pennsylvania State University, 525 Davey \\ Lab, University Park, PA 16802, USA \\ ${ }^{2}$ Departt of Astronomy, Columbia University, 550 West 120th Street, New York, NY 10027 \\ ${ }^{3}$ Instituto de Fisica, UFRGS, Campus do Vale, Porto Alegre, RS, Brazil \\ ${ }^{4}$ Dept. of Astronomy, University of California, Berkeley, CA 94720, USA \\ ${ }^{5}$ Dept. of Astronomy, University of Maryland, College Park, MD 20742, USA \\ ${ }^{6}$ Space Telescope Science Institute, 3700 San Martin Drive, Baltimore, MD 21218, USA
}

\begin{abstract}
We present the results of UV spectroscopy of AGNs with double-peaked Balmer emission lines. In $2 / 3$ of the objects, the far-UV resonance lines are strong, with single-peaked profiles resembling those of Seyfert galaxies. The Mg II line is the only UV line with a doublepeaked profile. In the remaining objects, the far-UV resonance lines are relatively weak but still single-peaked. The latter group also displays prominent UV absorption lines, indicative of a lowionization absorber. We interpret the difference in the profiles of the emission lines as resulting from two different regions: a dense, low-ionization accretion disk (the predominant source of the Balmer and Mg II lines), and a lower density, higher-ionization wind (the predominant source of the far-UV resonance lines). These results suggest a way of connecting the double-peaked emitters with the greater AGN population: in double-peaked emitters the accretion rate onto the black hole is low, making the wind feeble and allowing the lines from the underlying disk to shine through. This scenario also implies that in the majority of AGNs, the wind is the source of the broad emission lines.
\end{abstract}

\section{Introduction}

Broad, double-peaked Balmer lines are found in the spectra of approximately $20-25 \%$ of radio-loud AGNs at $z<0.4$ (Eracleous \& Halpern 1994, 2003) and in the spectra of $4 \%$ of all AGNs at $z<0.33$ from the Sloan Digital Sky Survey (Strateva et al. 2003). Half of these profiles are well described by kinematic models of axisymmetric, relativistic, Keplerian disks (Chen \& Halpern 1989; Chen et al. 1989), while the other half require a modified version of this model in which the disk is not axisymmetric (Eracleous et al. 1995; Strateva et al. 2003). The hosts of double-peaked emission lines (hereafter the "double-peaked emitters") share a number of distinguishing characteristics which make them stand out from the average AGN and suggest that these objects represent the extreme end of the distribution of AGN properties, namely: (a) Balmer line widths that are, on average, twice as large as those of other AGNs, (b) steep broad-line Balmer decrements, (c) unusually strong low-ionization emission lines (i.e., [O I] and [S II]), as well as unusually large [O I]/[O III] ratios, and (d) a great deal of starlight in the optical continuum. These characteristics support the scenario of Chen \& Halpern (1989) in which the inner accretion in these objects is an ion torus (Rees et al. 1982; known today as a radiatively inefficient accretion flow). Some of the spectroscopic properties of doublepeaked emitters are reminiscent of LINERs and the relation between the two groups is underscored by the detection of double-peaked Balmer lines in several LINERs. 


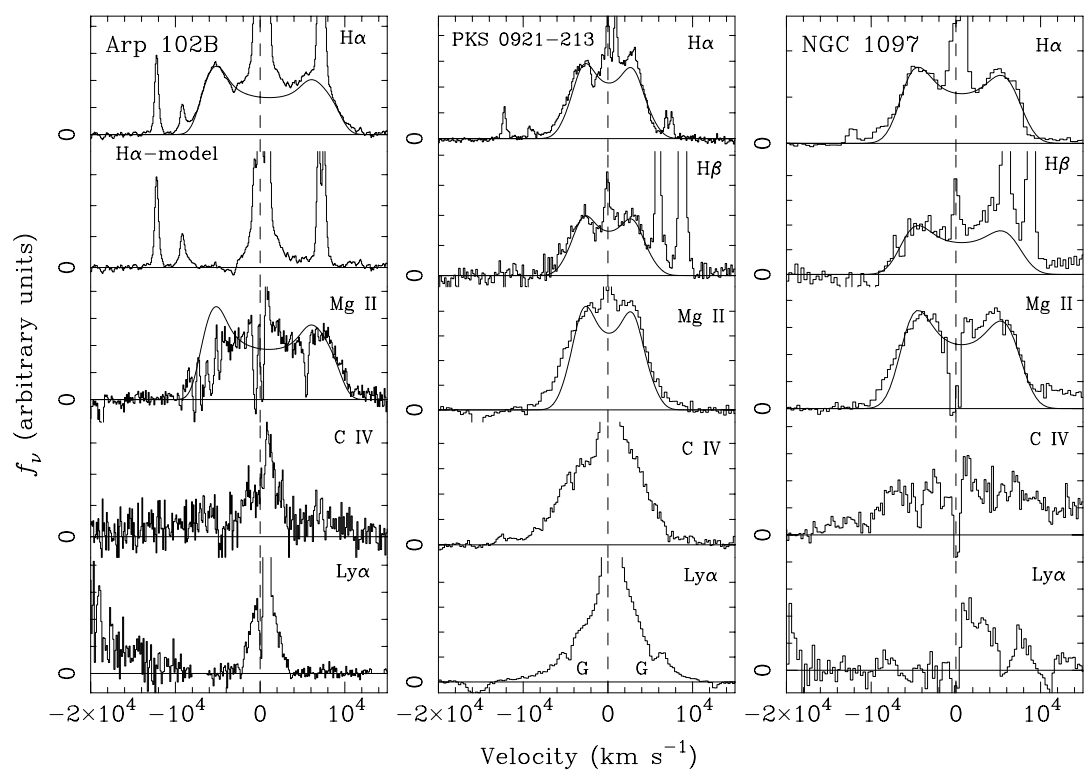

Figure 1. Optical, near-UV, and far-UV emission-line profiles of three double-peaked emitters. A circular-disk model is superposed on the Balmer and $\mathrm{Mg}$ II profiles for comparison. The $\mathrm{H} \alpha$ profile of Arp 102B after subtraction of the model is included, to show that the residual resembles the Ly $\alpha$ profile. Absorption lines marked with a "G" come from the gas in the Milky way, while all others arise in gas associated with the AGN.

\section{UV Spectroscopy with the Hubble Space Telescope and Interpretation}

UV spectra of several bright double-peaked emitters were observed with the HST's FOS and STIS instruments in order to compare the profiles of the high- and low-ionization UV emission lines with those of the Balmer lines, as well as to detect and study the shape of the UV continuum. The results of the observations of the prototypical object, Arp 102B, were presented and discussed by Halpern et al. (1996), while work on the other objects is in progress. A study of the spectral energy distribution of one of these objects, NGC 1097, is presented by Nemmen et al., in this volume.

The Mg II line is the only UV line with a double-peaked profile, while all the other UV lines are single-peaked. This is shown in Figure 1, where we compare the Balmer and UV line profiles of three representative objects. Particularly striking is the dramatic difference between the profiles of the Ly $\alpha$ and Balmer lines. In $2 / 3$ of the objects (exemplified by PKS 0921-213 in Fig. 1), the far-UV resonance lines are strong, with single-peaked profiles resembling those of Seyfert galaxies. In the remaining objects (e.g., Arp 102B and NGC 1097 in Fig. 1), the far-UV resonance lines are relatively weak but still singlepeaked. The emission-line profiles of Arp 102B also serve to illustrate that there are at least two broad-emission line regions in the same object. In particular, the profile of the $\mathrm{H} \alpha$ line can be decomposed into a very broad, double-peaked part which has no counterpart in the Ly $\alpha$ line, and a bell-shaped part of intermediate width, which closely resembles the Ly $\alpha$ profile. The double-peaked part can be attributed to emission from the dense, low-ionization gas in the accretion disk. The extremely weak Ly $\alpha$ emission from the disk was actually predicted by the models of weakly photoionized accretion disks by Collin-Souffrin \& Dumont (1989) and Dumont \& Collin-Souffrin (1990): at the 

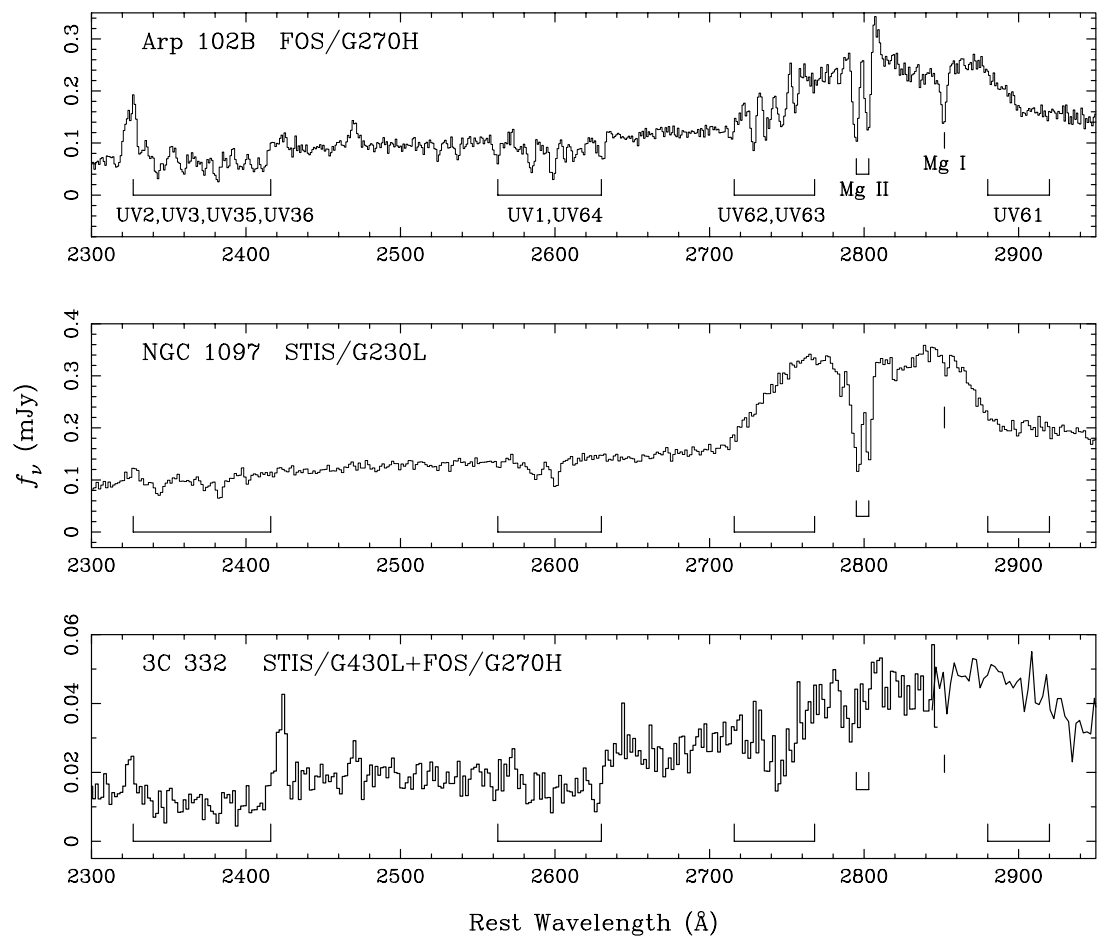

Figure 2. Comparison of the near-UV spectra of Arp 102B, 3C 332, and NGC 1097. All three objects have Fe II and Mg II absorption lines, although these lines seem to vary in strength from object to object. In particular, the UV62 and UV63 multiplets are noticeably absent in NGC 1097. These are also the objects that sport a weak UV continuum as well as weak far-UV emission lines.

high densities and column densities of the accretion disk Ly $\alpha$ photons are trapped by resonance scattering and the $n=2$ level of hydrogen is deexcited by collisions, thus the Ly $\alpha$ photons are effectively lost. On the other hand Balmer-line photons can escape relatively easily. The bell-shaped component can be ascribed to a medium of lower density and higher ionization, presumably a wind overlaying the disk. The propagation of the line photons through the accelerating wind results in a non-axisymmetric emissivity pattern as seen by the observer. Thus, the emission-line profiles from the wind are single-peaked, even though the dominant motion of the gas is rotational (Murray \& Chiang 1997).

We also discovered Fe II and Mg II associated absorption-line complexes in the UV spectra of some of the double-peaked emitters, as shown in Figure 2. Particularly interesting are the Fe II UV35-36 and UV61-64 multiplets, which arise from metastable lower levels located about $1 \mathrm{eV}$ above the ground state. The absorption lines are blueshifted by about $100-200 \mathrm{~km} \mathrm{~s}^{-1}$ relative to the systemic velocity, which suggests that they originate in an outflow. The case of Arp 102B was studied in great detail by Eracleous et al. (2003) who used X-ray observations to constrain the column density of the absorber and constructed photoionization models to investigate its structure. One of the main conclusions of that work was that the absorber is very likely to be a dense, low-ionization medium $\left(n \gtrsim 10^{11} \mathrm{~cm}^{-3}, 10^{-3.5}<U<10^{-2.5}\right.$ ), located just above the line-emitting portion of the accretion disk. Moreover, the absorber is likely to have the form of dense filaments, perhaps embedded in a lower-density, higher-ionization outflow. Thus the absorption lines trace an outflowing wind from the accretion disk, which is probably connected to the medium responsible for the single-peaked far-UV emission lines. 

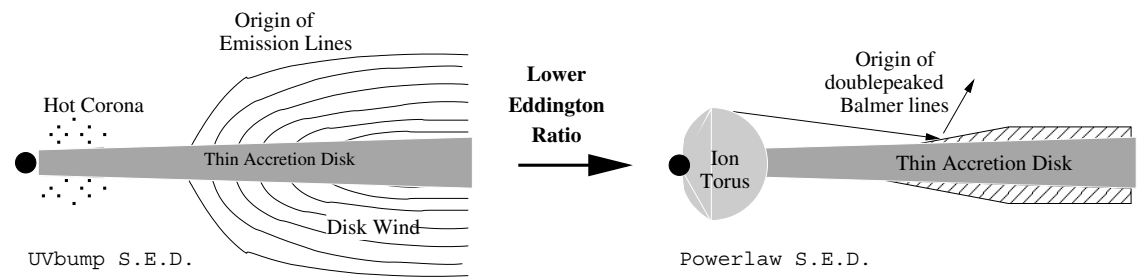

Figure 3. A sketch of how the structure of the accretion disk and its associated wind change as the Eddington ratio goes from high values $\left(\dot{M} / \dot{M}_{\text {Edd }} \gtrsim 0.1\right)$ to low values $\left(\dot{M} / \dot{M}_{\text {Edd }} \lesssim 10^{-3}\right)$. In the latter case the wind still exists but it is rather thin and inhomogeneous.

\section{Broader Implications}

The UV spectra of double-peaked emitters also lead to a number of general insights into AGN broad-line regions and they suggest a way of connecting double-peaked emitters with the greater AGN population. In this scheme, which is illustrated schematically in Figure 3, luminous AGNs (such as Seyfert galaxies and quasars) accrete at a rate that is a substantial fraction of the Eddington rate $\left(\dot{M} / \dot{M}_{\text {Edd }} \gtrsim 0.1\right)$. Thus they produce a significant wind, which is also the predominant source of broad emission lines (Murray et al. 1995; Proga et al. 2000). The double-peaked emitters, on the other hand, are the segment of the AGN population in which the accretion rate relative to the Eddington rate is extremely low $\left(\dot{M} / \dot{M}_{\mathrm{Edd}} \lesssim 10^{-3}\right)$. In this extreme, the inner accretion disk turns into an ion torus, the wind diminishes, and the accretion disk is unveiled.

We can exploit our direct view of the disk in double-peaked emitters to study its dynamics. This can be accomplished through long-term variability studies in which the variations of the double-peaked line profiles are compared with models of dynamical phenomena in the disk. Such studies require patience and persistence, as shown in the examples presented by Lewis et al. and Gezari et al. in this volume.

\section{Acknowledgements}

This work is supported by NASA through grant NAG5-10817 and grant GO-08684.01A from the Space Telescope Science Institute, which is operated by AURA, Inc., under NASA contract NAS5-26555.

\section{References}

Chen, K., \& Halpern, J. P. 1989, ApJ, 344, 115

Chen, K., Halpern, J. P., \& Filippenko, A. V. 1989, ApJ, 339, 742

Collin-Souffrin, S., \& Dumont, A. M. 1989, A\&A, 213, 29

Dumont, A. M. \& Collin-Souffrin, S. 1990, A\&A, 229, 313

Eracleous, M., \& Halpern, J. P. 1994, ApJS, 90, 1

Eracleous, M., \& Halpern, J. P. 2003, ApJ, 599, 886

Eracleous, M., Halpern, J. P., \& Charlton, J. C. 2003, ApJ, 582, 633

Eracleous, M., Livio, M., Halpern, J. P., \& Storchi-Bergmann, T. 1995, ApJ, 438, 610

Halpern, J. P., et al. 1996, ApJ, 467, 704

Murray, N., \& Chiang, J. 1997, ApJ, 474, 91

Murray, N., Chiang, J., Grossmann, S. A., \& Voit, G. M. 1995, ApJ, 451, 498

Proga, D., Stone, J. M., \& Kallman, T. R. 2000, ApJ, 543, 686

Rees, M. J., et al. 1982, Nature, 295, 17

Strateva, I. V., et al. 2003, AJ, 126, 1720 